Communications in Physics, Vol. 30, No. 4 (2020), pp. 363-372

DOI:10.15625/0868-3166/30/4/15152

\title{
PLASMONIC PHOTOTHERMAL HEATING OF GOLD NANOSHELL SOLUTIONS
}

\author{
DO THI NGA ${ }^{1}$, LE BA NAM ${ }^{2}$, TO THI THAO ${ }^{3}$ AND VU THI THUY DUONG ${ }^{4}$ \\ ${ }^{1}$ Institute of Physics, Vietnam Academy of Science and Technology \\ 10 Dao Tan, Ba Dinh, Hanoi, Vietnam \\ ${ }^{2}$ School of Engineering Physics, Hanoi University of Science and Technology \\ 1 Dai Co Viet, Hanoi, Vietnam \\ ${ }^{3}$ Department of Physics, Post and Telecommunications Institute of Technology \\ Nguyen Trai Street, Ha Dong District, Hanoi, Vietnam \\ ${ }^{4}$ Ministry of Science and Technology \\ 113 Tran Duy Hung Street, Cau Giay District, Hanoi, Vietnam \\ ${ }^{\dagger} E$-mail: dtnga@iop.vast.ac.vn \\ Received 18 June 2020 \\ Accepted for publication 25 October 2020
}

Published 16 December 2020

\begin{abstract}
We present a theoretical approach to investigate how gold core-shell nanoparticles dispersed in water thermally respond to near-infrared-regime laser illumination. First, the absorption cross section of gold nanostructures is calculated using Mie theory. Second, we determine the absorbed power density when the solution is exposed by the laser light. Here, the solution of nanostructures is assumed to be uniform and homogenous. Third, we analytically solve the transient bioheat equation to calculate time-dependent temperature increase. Our theoretical calculations quantitatively agree with previous experiments without any adjustable parameter. This approach would provide a reliable tool to understand photothermal effects in unexplored structures.
\end{abstract}

Keywords: photothermal effects, gold nanoshells, NIR, Mie theory.

Classification numbers: 78.20.nb, 78.67.Bf. 
DO THI NGA et al.

\section{INTRODUCTION}

Photothermal effects of gold nanoshells have been recently investigated due to their applications in a wide range of fields [1-6]. Core-shell nanoparticles are composed of two different materials to exploit their advantageous features and shift surface plasmon resonance in near-infrared regime, where water absorbs a very small amount of optical energy. Then, one can maximize the energy harvesting of gold nanostructures from incident laser light to heat up the solution. When injected into biological samples, gold nanostructures can locally and selectively heat and eradicate unhealthy cells without damaging healthy counterparts $[7,8]$.

Moreover, the photo-induced heat generation of gold nanoparticles leads to acoustic waves and significantly enhances a new depth optoacoustic imaging $[9,10]$. An increase of efficiency of the surface plasmon absorption generates the enhancement of the ultrasonic emission of the contrast. Therefore, the resolution of this image is significantly increased and can be applied for non-invasive diagnostic techniques. Gold nanoparticles can also penetrate cell membranes $[7,8]$ and are one of the most promising drug delivery vehicles.

However, applications of gold nanoshells are strongly dependent on particle sizes. For example, effective drug delivery [11-13] requires sub-100-nm nanoparticles. However, when the diameter of gold nanoparticles is greater than $100 \mathrm{~nm}$, it is hard to penetrate membranes and these large particles can be adsorbed on blood vessels and biological organs [12]. Thus, investigating optical properties and photothermal effects of nanoparticles in different sizes is essential for real applications.

In this paper, we show a simple but comprehensive model to calculate the spatial and temporal temperature under laser exposure of gold-nanoshell aqueous solutions. Finite-size and structural effects of nanostructures on the absorbed optical energy are studied. To verify our theoretical approach, we compare numerical results with experimental data. Finally, we discuss limitations of this approach and possibilities to develop.

\section{MIE THEORY FOR GOLD CORE-SHELL NANOSTRUCTURE}

The absorption, scattering, and extinction cross sections of core-shell nanospheres have been theoretically calculated using Mie theory [3,14-19]. It is well-known that theoretical calculations provide a quantitatively good agreement with experiments $[15-17,19]$. These cross sections are

$$
\begin{aligned}
Q_{e x t} & =-\frac{2 \pi}{k_{m}^{2}} \sum_{n=1}^{\infty}(2 n+1) \operatorname{Re}\left(a_{n}+b_{n}\right), \\
Q_{s c a t} & =\frac{2 \pi}{k_{m}^{2}} \sum_{n=1}^{\infty}(2 n+1)\left(\left|a_{n}\right|^{2}+\left|b_{n}\right|^{2}\right), \\
Q_{a b s} & =Q_{e x t}-Q_{\text {scat }},
\end{aligned}
$$

where

$$
a_{n}=-\frac{U_{n}^{T M}}{U_{n}^{T M}+i V_{n}^{T M}}, \quad b_{n}=-\frac{U_{n}^{T E}}{U_{n}^{T E}+i V_{n}^{T E}},
$$




$$
\begin{aligned}
U_{n}^{T M} & =\left|\begin{array}{cccc}
j_{n}\left(k_{c} R_{c}\right) & j_{n}\left(k_{s} R_{c}\right) & y_{n}\left(k_{s} R_{c}\right) & 0 \\
\Psi_{n}^{\prime}\left(k_{c} R_{c}\right) & \frac{\Psi_{n}^{\prime}\left(k_{s} R_{c}\right)}{\varepsilon_{c}} & \frac{\Phi_{n}^{\prime}\left(k_{s} R_{c}\right)}{\varepsilon_{s}} & 0 \\
0 & j_{n}\left(k_{s} R_{s}\right) & y_{n}\left(k_{s} R_{s}\right) & j_{n}\left(k_{m} R_{s}\right) \\
0 & \frac{\Psi_{n}^{\prime}\left(k_{s} R_{s}\right)}{\varepsilon_{c}} & \frac{\Phi_{n}^{\prime}\left(k_{s} R_{s}\right)}{\varepsilon_{s}} & \frac{\Psi_{n}^{\prime}\left(k_{m} R_{s}\right)}{\varepsilon_{m}}
\end{array}\right|, \\
V_{n}^{T M} & =\left|\begin{array}{cccc}
j_{n}\left(k_{c} R_{c}\right) & j_{n}\left(k_{s} R_{c}\right) & y_{n}\left(k_{s} R_{c}\right) & 0 \\
\frac{\Psi_{n}^{\prime}\left(k_{c} R_{c}\right)}{\varepsilon_{c}} & \frac{\Psi_{n}^{\prime}\left(k_{s} R_{c}\right)}{\varepsilon_{s}} & \frac{\Phi_{n}^{\prime}\left(k_{s} R_{c}\right)}{\varepsilon_{s}} & 0 \\
0 & j_{n}\left(k_{s} R_{s}\right) & y_{n}\left(k_{s} R_{s}\right) & y_{n}\left(k_{m} R_{s}\right) \\
0 & \frac{\Psi_{n}^{\prime}\left(k_{s} R_{s}\right)}{\varepsilon_{c}} & \frac{\Phi_{n}^{\prime}\left(k_{s} R_{s}\right)}{\varepsilon_{s}} & \frac{\Phi_{n}^{\prime}\left(k_{m} R_{s}\right)}{\varepsilon_{m}}
\end{array}\right|,
\end{aligned}
$$

where $R_{c}$ and $R_{s}$ are the inner and outer radius of the core-shell nanostructure, respectively, and $V_{n}$ and $U_{n}$ are determinants, $j_{n}(x)$ is the spherical Bessel function of the first kind, $y_{n}(x)$ is the spherical Neumann function, $\Psi(x)=x j_{n}(x)$ and $\xi_{n}(x)=x y_{n}(x)$ are the Riccati-Bessel functions. One can obtain analytical expressions of $U_{n}^{T E}$ and $V_{n}^{T E}$ by replacing the dielectric function with the permeability in Eq.(2). The dielectric functions of the core and shell of the core-shell nanoparticles are $\varepsilon_{c}$ and $\varepsilon_{s}$, respectively. The dielectric constant of surrounding (water) medium is $\varepsilon_{m} \approx 1.77$. The wavenumber is $k_{i}=2 \pi \sqrt{\varepsilon_{i}} / \lambda$ with $i=s, c$, and $m$ with $\lambda$ being the wavelength of incident light.

In our work, we study the optical spectra of silica-gold nanoshells. The dielectric constant of silica is $\varepsilon_{c} \approx 2.13$ [20]. In order to simplify calculations, we choose the Lorentz-Drude model with many oscillators to describe the dielectric function of gold $\left(\varepsilon_{S}\right.$ or $\left.\varepsilon_{A u}\right)$ [21], which is

$$
\varepsilon_{A u}=\varepsilon_{s}=1-\frac{f \omega_{p}^{2}}{\omega^{2}-i \omega \Gamma_{0}}+\sum_{j=1}^{5} \frac{f_{j} \omega_{p}^{2}}{\omega_{j}^{2}-\omega^{2}+i \omega \Gamma_{j}},
$$

where $\omega_{p}$ is the gold plasma frequency, $\Gamma_{0}$ and $\Gamma_{j}$ are the gold damping parameters, and $f_{0}$ and $f_{j}$ are the gold oscillator strengths. Values of these parameters are taken from Ref. [21]. In several experimental studies $[3,15,19]$, the shell thickness is approximately $20 \mathrm{~nm}$ and the finite-size effect on the optical spectrum becomes important. Hereafter, we fix the thickness $R_{s}-R_{c}=20 \mathrm{~nm}$ to be consistent with Ref. $[3,15,19]$. This finite-size effect is encoded to our dielectric function by changing the damping parameter $\Gamma_{0} \equiv \Gamma_{0}+B v_{F} /\left(R_{s}-R_{c}\right)$, where $v_{F}$ is the gold Fermi velocity and $B$ is a parameter responsible for the scattering processes. An increase of $B$ not only shifts a position of the plasmonic resonance, but also broadens optical sepectra. The broadening of optical spectra contains information of the size distribution of nanostructures in experiments. For silica-gold core-shell nanoparticles, the parameter $B$ is fixed at 1.5 [17,22].

Figure 1 shows the theoretical absorption spectra calculated using Mie theory at $R_{c}=10$, $20,30,40,50$, and $60 \mathrm{~nm}$. Increasing the silica core size leads to an increase of the absorption cross section and a red-shift of the plasmonic resonance. These peaks are located at $\lambda_{\max } \approx 541$ $\mathrm{nm}$ and $782 \mathrm{~nm}$ for $R_{c}=10 \mathrm{~nm}$ and $60 \mathrm{~nm}$, respectively. As shown in Fig. 2, increasing the core size $R_{c}$ leads to a growth of the plasmonic resonance position $\lambda_{\max }$. The linear guide-to-the-eye 


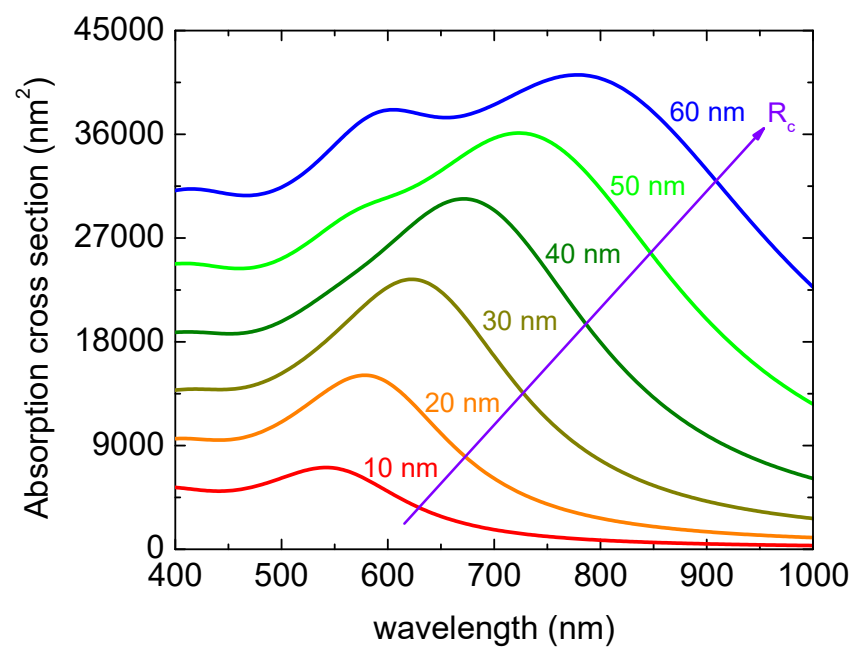

Fig. 1. (Color online) Theoretical absorption cross section of silica-gold nanoshells as a function of wavelength at different sizes of silica core. The gold thickness is fixed at $20 \mathrm{~nm}$.

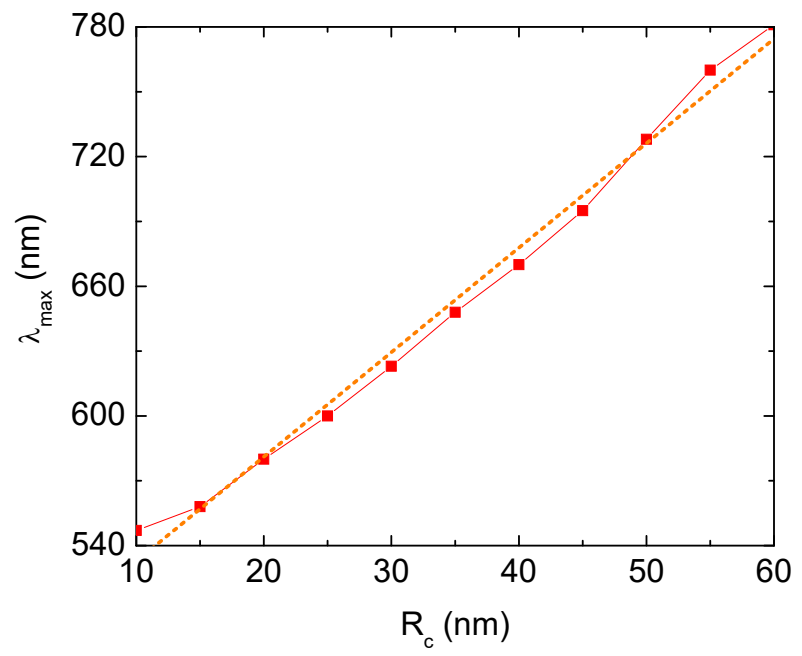

Fig. 2. (Color online) Position of surface plasmon resonance (SPR) as a function of radius of the silica core radius. The solid and dotted lines are our full calculations and a guide-to-the-eye line, respectively.

line is found to be $\lambda_{\max } \approx 484.44+4.8 R_{c}$. This linearity of the guide-to-the-eye line is consistent with prior works $[14,23,24]$. To exploit photothermal effects of gold nanoshells in biological applications, the core radius is greater than $40 \mathrm{~nm}$. 
When $R_{c} \geq 40 \mathrm{~nm}$, one can see a shoulder in the optical spectrum of gold nanoshell. This emergence is attributed to the contribution of higher-order multipoles (for example, dipolemultipole and multipole-multipole interactions) to the absorption process [14]. For smaller nanostructures, the role of the silica core is screened and the composite can be considered as a solid gold nanosphere. Electromagnetic fields penetrating into the gold layer can be coupled in the silica core. However, the plasmonic coupling is decoupled when the core size is large or the dielectric region is sufficiently large to decouple the interference of electric fields in the metal shell.

\section{PLASMONIC PHOTOTHERMAL HEATING}

In biological applications of photothermal effects, near-infrared laser light is preferred since it can penetrate into water and biological tissue without large energy loss to excite surface plasmon resonances of metal nanostructures. To be consistent with prior experiments [25], in our calculations, we choose the $808 \mathrm{~nm}$ laser light. It is important to note that this laser light has been widely used in other studies [3, 15, 19].

Plasmonic nanostructures absorb optical energy of incident light and thermally dissipate into the solution. Thus, the temperature of the solution is increased under laser illumination. To model physically the heating process, we have three assumptions: (1) $100 \%$ of the absorbed energy can be converted to the heat; (2) plasmonic particles in the solution moves freely and randomly; (3) to solve analytically the heat transfer equation in the easy manner, we adopt spherical coordinates for the equation, and this solution with a volume $V$ is described as an effective spherical solution of radius $R$. Thus, $R=(3 V / 4 \pi)^{1 / 3}$.

Under the laser light irradiation, the heating process is mainly governed by two tightly intercorrelated mechanisms: light-to-heat conversion of particles and thermal convection in medium. By adopting the Pennes bioheat transfer equation $[15,26]$ in spherical coordinates, we have the time-dependent temperature increase $\Delta T(r, t)$ expressed by

$$
\frac{1}{\kappa} \frac{\partial \Delta T}{\partial t}=\frac{1}{r^{2}} \frac{\partial}{\partial r}\left(r^{2} \frac{\partial \Delta T}{\partial r}\right)-\frac{\Delta T}{\kappa \tau}+\frac{A}{k}
$$

where $k$ and $\kappa=k /(\rho c)$ are the thermal conductivity and thermal diffusivity of the medium, respectively. $\rho=1000 \mathrm{~kg} / \mathrm{m}^{3}$ is the mass density, $c=4200 \mathrm{~J} /(\mathrm{kg} . \mathrm{K})$ is the specific heat, and $\tau$ is the perfusion time constant. $A=N Q_{a b s} I_{0}$ is the heat source density calculated by summing all absorbed energy of each plasmonic nanoparticles. Here, $N$ is the density number of particles in experimental samples and $I_{0}$ is the laser intensity. The sum of the absorbed energy of each nanoshell implies that there is no plasmonic coupling or aggregation among nanostructures.

Since the heat source generation is only confined within the effective spherical region and there is no nanoparticle to absorb and generate heat outside this area, thus, one can rewrite Eq. (4) to be

$$
\begin{aligned}
& \frac{1}{\kappa} \frac{\partial \Delta T}{\partial t}=\frac{1}{r^{2}} \frac{\partial}{\partial r}\left(r^{2} \frac{\partial \Delta T}{\partial r}\right)-\frac{\Delta T}{\kappa \tau}+\frac{A}{k}, \quad 0 \leq r \leq R, \\
& \frac{1}{\kappa} \frac{\partial \Delta T}{\partial t}=\frac{1}{r^{2}} \frac{\partial}{\partial r}\left(r^{2} \frac{\partial \Delta T}{\partial r}\right)-\frac{\Delta T}{\kappa \tau}, \quad R \leq r .
\end{aligned}
$$

Solving analytically Eq.(5) requires the continuous boundary conditions. These are continuities of the spatial temperature increase and its spatial derivatives at $r=R$. The temperature 
$\Delta T(r=0, t)$ of the theoretical spherical domain is supposed to be the temperature experimentally probed by thermocouples/thermalsensor $[15,26]$. The measured temporal temperature is

$$
\begin{aligned}
\Delta T(r=0, t) & =\frac{A}{k}\left[-\kappa \int_{0}^{t} e^{-t^{\prime} / \tau} \operatorname{erfc}\left(\frac{R}{2 \sqrt{\kappa t^{\prime}}}\right) d t^{\prime}\right. \\
& -R \int_{0}^{t} e^{-t^{\prime} / \tau} \sqrt{\frac{\kappa}{\pi t^{\prime}}} \exp \left(-\frac{R^{2}}{4 \kappa t^{\prime}}\right) d t^{\prime} \\
& \left.+\kappa \tau\left(1-e^{-t / \tau}\right)\right] .
\end{aligned}
$$

\section{NUMERICAL RESULTS AND DISCUSSIONS}

To validate our approach, theoretical calculations are compared to prior experiments [25], which investigated photothermal effects of gold nanoshells having $R_{c}=60 \mathrm{~nm}$ and $R_{s}=80 \mathrm{~nm}$ dispersed in water. In these experiments, the volume of the solution is $V=260 \mu 1$, the laser intensity is $I_{0}=2 \mathrm{~W} / \mathrm{cm}^{2}$.

Figure 3 shows theoretical results and experimental data in Ref. [25] for the time-dependent temperature rise of the gold-nanoshell solution at different concentrations $\left(1.14 \times 10^{9} \mathrm{particles} / \mathrm{ml}\right.$ and $2.28 \times 10^{9}$ particles $/ \mathrm{ml}$ ). Our Mie calculation gives $Q_{a b s}=40800 \mathrm{~nm}^{2}$ at $808 \mathrm{~nm}$ to calculate the heat source power. For clean water, the perfusion time is infinite or very large since the convective heat transfer of water is much smaller than that of solution having biological components due to a lack of the circulatory system. In other biological systems such as chicken tissues [15], $\tau=200 \mathrm{~s}$. A good agreement between numerical results for $\tau \geq 2000 \mathrm{~s}$ and experiments verify the purity of water. A decrease of the perfusion time leads to a faster saturation of the temperature rise and also reduces the steady-state temperature. This finding indicates an important role of perfusion in the heating process in biosystems. Investigating photothermal effects in these systems without considering the perfusion mechanism causes a significant deviation.

It is important to note that, for $\tau=\infty$, our theoretical curve deviates from experimental data, particularly when $t \geq 60 \mathrm{~s}$. There are several possible reasons for this deviation. First, we ignore the temperature-induced interfacial change and thermal instability [27]. Second, a temperature increase also modifies parameters in dielectric functions [28, 29]. In Ref. [27, 28], when $\Delta T \geq 100{ }^{0} \mathrm{C}$, effects of temperature on optical and structural properties become significant. Although our temperature rise is less than $20{ }^{\circ} \mathrm{C}$, the heating process possibly shifts the absorption spectrum. Third, the thermal conductivity is temperature-dependent $[30,31]$ but it is assumed to be unchanged in our model for simplification purpose.

Equation (6) suggests that the steady-state temperature linearly increases with the laser intensity $I_{0}$ and the density number of nanoparticles. This analysis is in accordance with experimental findings and other theoretical approaches $[3,4,15,32,33]$. However, theoretical predictions can deviate from experiments when the solution of gold nanoshells is very dense since plasmonic scattering effects become more important and the light-to-heat conversion is not perfect. Note that the scattering cross section is much less than the absorption counterpart for small particles [14] and this role is reversed at large particles. In addition, plasmonic couplings between nanostructures may occur and invalidate our assumptions. From practical point of view, the plasmonic coupling effects occur in the dense solution of nanostructures and they definitely damage body. 

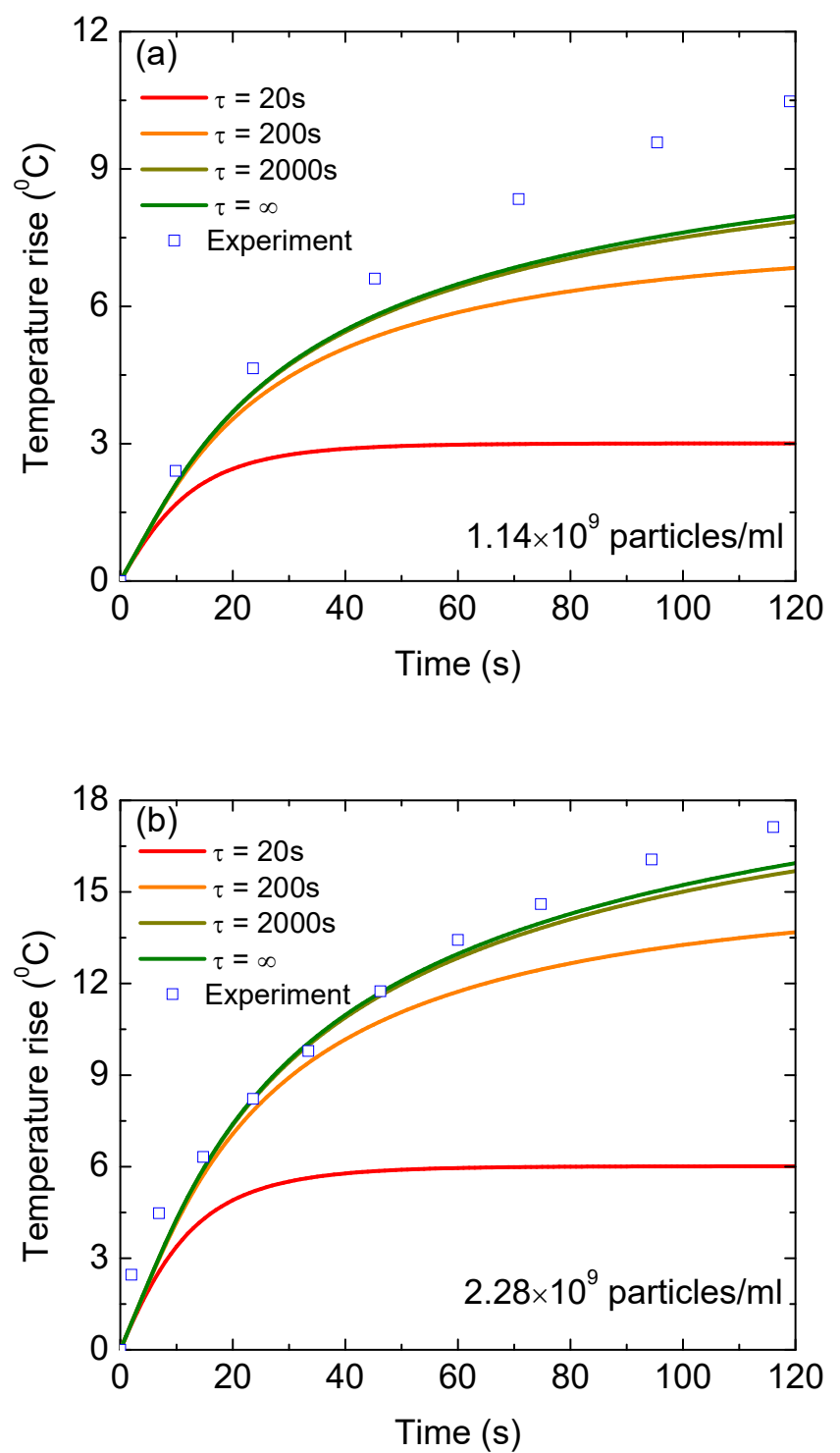

Fig. 3. (Color online) Theoretical (solid lines) and experimental (open points) temperature rise for a solution of nanoshells with $20 \mathrm{~nm}$ gold thickness on a silica core with a radius of $60 \mathrm{~nm}$ at a concentration of (a) $1.14 \times 10^{9}$ particles $/ \mathrm{ml}$ and (b) $2.28 \times 10^{9}$ particles $/ \mathrm{ml}$ to describe experiments in Ref. [25]. The perfusion time ranges from 20 seconds to infinity. The thermal conductivity is $k=0.6 \mathrm{~W} / \mathrm{m} . \mathrm{K}$. 
Thus, in most cases, the nanostructure solution is dilute. Revealing underlying mechanisms and main reasons for this deviation requires systematic future experiments.

Figure 4 shows the time dependence of the temperature increase calculated by our model for the solution of gold nanoshells having $1.14 \times 10^{9}$ particles $/ \mathrm{ml}$ and $\tau=2000 \mathrm{~s}$ at several values of the thermal conductivity. The higher thermal conductivity accelerates the heat transfer process and thus reduces $\Delta T(r=0, t)$. An addition of gold nanoparticles to the solution enhances this conductivity. However, the enhancement is neglectable at dilute solutions and this is one of limitations in our approach.

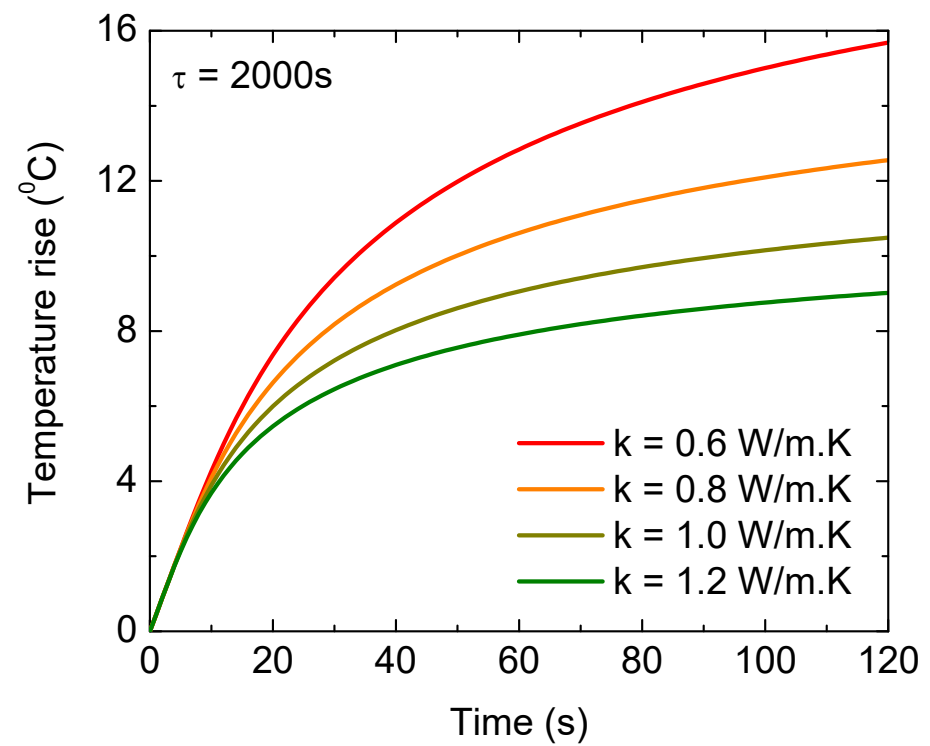

Fig. 4. (Color online) Theoretical temperature rise for a solution of nanoshells with 20 $\mathrm{nm}$ gold thickness on a silica core with a radius of $60 \mathrm{~nm}$ at different values of the thermal conductivity. The perfusion time is $2000 \mathrm{~s}$. The particle concentration is $2.28 \times 10^{9}$ particles $/ \mathrm{ml}$.

In Eqs. (4) and (6), one can see that the product $\kappa \tau$ plays an important role in the time dependence of the temperature rise. However, since biological tissues are mostly covered by water $(\sim 90 \%)$ [15], the thermal conductivity and thermal diffusitivity of these systems are approximately equal to those of water [15]. Thus, in photothermal therapy investigations, roles of the perfusion time become dominant and crucial for predicting an increase of temperature. In addition, by tuning the perfusion time to obtain the best fit between theory and experiment, we can determine the value of the perfusion time. 


\section{CONCLUSIONS}

In summary, we have presented the theoretical approach to calculate the time-dependent temperature rise of gold nanoshells dispersed in water under laser illumination. We calculate the absorption cross section of these nanostructures by the scattering Mie theory. The solution of gold nanoshells is dilute to ignore plasmonic coupling and scattering effects. This assumption allows us to calculate the absorbed optical energy on a particle and the power source density in the solution. The absorbed energy of near-infrared light at $808 \mathrm{~nm}$ on nanoparticles thermally dissipates into medium to heat up the system. By solving the Pennes bioheat transfer equation, we obtain an analytical expression of the temperature rise. The analysis indicates a linear increase of the steady-state temperature with increasing the laser intensity and nanostructure concentration. The perfusion effects are very important in the laser-induced heating process in biological systems. Our calculations quantitatively agree well with various experimental studies. Moreover, photothermal effects are suppressed at systems having the high thermal conductivity. This approach can be applied for other various nanostructures and be used to determine the perfusion time of biological systems. Limitations of this theoretical approach are also discussed.

\section{ACKNOWLEDGMENT}

This research is funded by Vietnam National Foundation for Science and Technology Development (NAFOSTED) under grant number 103.01-2018.337.

\section{REFERENCES}

[1] Mark L. Brongersma, Nat. Mater. 2 (2003) 296.

[2] J.-F. Li, Y.-J. Zhang, S.-Y. Ding, R. Panneerselvam and Z.-Q. Tian, Chem. Rev. 117 (2017) 5002.

[3] H. H. Richardson, Z. N. Hickman, A. O. Govorov, A. C. Thomas, W. Zhang and M. E. Kordesch, Nano Lett. 6 (2006) 783.

[4] H. H. Richardson, M. T. Carlson, P. J. Tandler, P. Hernandez and A. O. Govorov, Nano Lett. 9 (2009) 1139.

[5] A. Abbasi, K. Park, A. Bose and G. D. Bothun, Langmuir 33 (2017) 5321.

[6] A. F. Zedan, S. Moussa, J. Terner, G. Atkinson and M. S. El-Shall, ACS Nano 7 (2013) 627.

[7] F. Simonelli, D. Bochicchio, R. Ferrando and G. Rossi, J. Phys. Chem. Lett. 6 (2015) 3175.

[8] A. Verma, O. Uzun, Y. Hu, Y. Hu, H.-S. Han, N. Watson, S. Chen, D. J. Irvine and F. Stellacci, Nat. Mater. 7 (2008) 588.

[9] Y.-S. Chen, W.Frey, S. Kim, P. Kruizinga, K. Homan and S. Emelianov, Nano Lett. 11 (2011) 348.

[10] J. D. Dove, T. W. Murray and M. A. Borden, Soft Matters 9 (2013) 7743.

[11] W. Sun, Q. Hu, W. Ji, G. Wright and Z. Gu, Physiol. Rev. 97 (2017) 189.

[12] D. T. Wiley, P. Webster, A. Gale and M. E. Davis, Proc. Natl. Acad. Sci. USA 110 (2013) 8662.

[13] E. Blanco, H. Shen and M. Ferrari, Nat. Biotechnol. 33 (2015) 941.

[14] Michael Quinten, Optical properties of nanoparticle systems, Wiley, Weinheim, Germany (2011).

[15] V. T. T. Duong, A. D. Phan, N. T. H. Lien, D. T. Hue, D. Q. Hoa, D. T. Nga, T. H. Nhung and Nguyen A. Viet, Phys. Status Solidi A 215 (2018) 1700564.

[16] A. D. Phan, N. B. Le, N. T. H. Lien and K. Wakabayashi, J. Phys. Chem. C 122 (2018) 19801.

[17] A. D. Phan, D. T. Nga and N. A. Viet, Opt. Commun. 410 (2018) 108.

[18] J.-Y. Lee, M.-C. Tsai, P.-C. Chen, T.-T. Chen, K.-L. Chan, C.-Y. Lee and R.-K. Lee, J. Phys. Chem. C 119 (2015) 25754.

[19] A. D. Phan, N. C. Do and D. T. Nga, J. Phys. Soc. Jpn. 86 (2017) 084401.

[20] Edward D. Palik, Handbook of Optical Constants of Solids, Academic, New York (1985).

[21] A. D. Rakic, A. B. Djurisic, J. M. Elazar and M. L. Majewski, Appl. Opt. 37 (1998) 5271. 
[22] Z. Qin, Y. Wang, J. Randrianalisoa, V. Raeesi, W. C.W. Chan, W. Lipinski and J. C. Bischof, Sci. Rep. 6 (2016) 29836.

[23] R. Wannemacher, A. Pack and M. Quinten, Appl. Phys. B 68 (1999) 225.

[24] A. Campos, N. Troc, E. Cottancin, M. Pellarin, H.-C. Weissker, J. Lerme, M. Kociak and M. Hillenkamp, Nat. Phys. 15 (2019) 275.

[25] V. P. Pattani and J. W. Tunnell, Laser Surg. Med. 44 (2012) 675.

[26] S. J. Norton and T. Vo-Dinh, J. Appl. Phys. 119 (2016) 083105.

[27] K. Zhang, W. Wang, W. Cheng, X. Xing, G. Mo, Q. Cai, Z. Chen and Z. Wu, J. Phys. Chem. C 114 (2010) 41.

[28] N. Daneshfar, Phys. Plasmas 21 (2014) 063301.

[29] S. Link and M. A. El-Sayed, J. Phys. Chem. B 103 (1999) 4212.

[30] L. Yu-Hua, Q. Wei and F. Jian-Chao, Chin. Phys. Lett. 25 (2008) 3319.

[31] H. Angue Mintsa, G. Roy, C. T. Nguyen and D. Doucet, Int. J. Therm. Sci. 48 (2009) 363.

[32] F. Wang, Y. Huang, Z. Chai, M. Zeng, Q. Li, Y. Wanga and D. Xu, Chem. Sci. 7 (2016) 6887.

[33] A. D. Phan, D. T. Nga, D. C. Nghia, V. D. Lam and K. Wakabayashi, Phys. Status Solidi RRL 14 (2020) 1900656. 